\title{
Diagnosis and treatment of constrictive bronchiolitis
}

\section{Gary R Epler}

\author{
Address: Pulmonary and Critical Care Medicine, Brigham and Women's Hospital, Harvard Medical School, 75 Francis Street, Boston, \\ MA 02115, USA \\ Email: gepler@partners.org
}

FI000 Medicine Reports 2010, 2:32 (doi:10.3410/M2-32)

The electronic version of this article is the complete one and can be found at: http://fl000.com/reports/medicine/content/2/32

\begin{abstract}
Constrictive bronchiolitis is a bronchiolar airway disease that surrounds the lumen with fibrotic concentric narrowing and obliteration. The mosaic pattern seen on the expiratory high-resolution chest CT scan is diagnostic in an individual with shortness of breath, early inspiratory crackles, and irreversible airflow obstruction. Swyer-James-MacLeod syndrome is no longer considered a congenital disorder but as constrictive bronchiolitis detected in young adults who had infectious pneumonia during infancy. For lung transplant recipients, tacrolimus continues to be an important immune suppression medication, extracorporeal photopheresis may improve the decline of pulmonary function, and azithromycin may be effective in some lung transplant recipients for treatment of bronchiolitis obliterans syndrome for prevention of constrictive bronchiolitis.
\end{abstract}

\section{Introduction and context}

Constrictive bronchiolitis is a small airway fibrotic respiratory disease. Clinicians use the term constrictive bronchiolitis, bronchiolitis obliterans, or obliterative bronchiolitis. The pathologist uses the term constrictive bronchiolitis and will report the lesion with or without airway obliteration. The clinically significant disease is associated with the fibrotic obliteration of the bronchiolar airways. This fibrotic constrictive lesion develops externally to the airway lumen, constricting the airway in a concentric manner with eventual obliteration of the lumen. This is distinct from the inflammatory proliferative lesion that develops internally from the airway wall, filling the lumen with an inflammatory polypoid lesion or buds of granulation tissue, and referred to as proliferative bronchiolitis. The fibrotic constrictive bronchiolitis involves the mid-bronchiole region. The inflammatory proliferative bronchiolitis lesion may involve the mid-bronchiole region, but usually involves the distal bronchioles extending into alveolar spaces, and is referred to as bronchiolitis obliterans organizing pneumonia (BOOP) [1].

Constrictive bronchiolitis is a peribronchiolar fibrotic process that surrounds, rather than fills, the lumen with concentric narrowing and obliteration. The lesion preferentially involves membranous bronchioles and spares distal respiratory bronchioles. Constrictive bronchiolitis may be patchy, making the diagnosis difficult from a transbronchial biopsy. The diagnosis is also difficult in regions showing no bronchiolar inflammation and in completely scarred, distorted bronchioles.

Shortness of breath is the most common presenting symptom, although cough may occur early. Chest physical examination may be normal or show early inspiratory crackles or inspiratory 'squeaks'. The chest X-ray is often normal or shows hyperinflation. Inspiratory and expiratory chest computed tomography (CT) scans can be diagnostic in an appropriate setting, such as in an individual who is a non-smoker without asthma. The expiratory high-resolution chest CT (HRCT) scan shows a 'mosaic' pattern, which is a patchwork of high- and lowdensity regions resulting from air-trapping by constricted and partially obliterated bronchioles (Figure 1). Bronchiectasis and bronchiolectasis represent severe scarring and are late findings. Pulmonary function testing typically shows irreversible airflow obstruction as measured by a decrease in the forced expired volume in 1 second 
Figure I. Inspiratory and expiratory high-resolution chest CT scans showing the mosaic pattern diagnostic of constrictive bronchiolitis

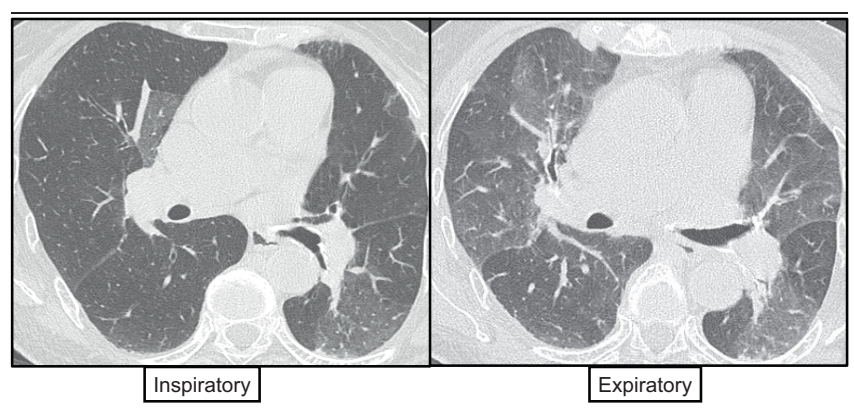

The scans are from a bone-marrow recipient who had shortness of breath and an FEV (forced expired volume in I second) that revealed a severe airflow obstruction. The chest X-ray was normal. The inspiratory HRCT scan on the left shows mostly normal lung; however, the expiratory HRCT scan on the right shows a mosaic pattern with regions of air trapping from constricted and partially obliterated bronchioles, consistent with constrictive bronchiolitis. The scans were gratefully provided by Dr Ritu Gill, Chest Radiology, Brigham and Women's Hospital, Boston, MA, USA.

$\left(\mathrm{FEV}_{1}\right)$ or the $\mathrm{FEV}_{1} / \mathrm{FVC}$ (forced vital capacity) ratio. In rare situations, a restrictive pattern may be seen, usually caused by dynamic compression of the lungs caused by airtrapping. The diffusing capacity may be normal or decreased.

Constrictive bronchiolitis may either be a primary lesion or a lesion associated with other lung diseases or systemic disorders. Idiopathic constrictive bronchiolitis is very rare. Toxic fume constrictive bronchiolitis continues to be reported intermittently, recently from mustard gas exposure and among food-flavoring workers using the ketone butter flavoring diacetyl. The diagnosis continues to be established using the 'three-phase' model of the illness, beginning with an asymptomatic latency period of a few hours after the exposure. The second, acute phase is characterized by severe shortness of breath and pulmonary edema in the form of acute respiratory distress syndrome often requiring mechanical ventilation in the intensive care unit. This acute phase is treated with massive doses of intravenous corticosteroid therapy. After resolution, the third phase begins as an asymptomatic latency period of several days followed by constrictive bronchiolitis with irreversible airflow obstruction. After completion of a course of corticosteroids, this final phase of the illness is managed by an active pulmonary rehabilitation program and a home exercise program.

Constrictive bronchiolitis may occur from aspiration. An unusual cause of deadly constrictive bronchiolitis has been reported among women consuming juice from a leafy Malaysian vegetable, Sauropus androgynus, which is purchased from street vendors and used for weight control. Post-infection constrictive bronchiolitis may develop after viral pneumonia or Mycoplasma pneumonia. Constrictive bronchiolitis caused by drugs is rare and limited to penicillamine, gold, and possibly 5-fluorouracil. Connective-tissue-related constrictive bronchiolitis occurring among individuals with rheumatoid arthritis and scleroderma is rare, but may cause disabling respiratory symptoms. Constrictive bronchiolitis has been reported in patients with inflammatory bowel disease (caused by ulcerative colitis or Crohn's disease), Stevens-Johnson syndrome, paraneoplastic autoimmune multi-organ syndrome, and neuroendocrine cell hyperplasia. Constrictive bronchiolitis may occur in allogeneic marrow transplantation recipients and is preceded by chronic graft-versus-host disease (GVHD). Constrictive bronchiolitis as a result of stem cell transplantation may occur in a small percentage of patients. Post-lung transplantation constrictive bronchiolitis continues to be a major life-threatening complication, affecting up to $50-60 \%$ of patients who survive five years after transplantation. A non-biopsy classification for bronchiolitis obliterans syndrome (BOS) using, amongst other parameters, various levels of $\mathrm{FEV}_{1}$ values has been established as a useful clinical designation. This is a clinical classification that may include both proliferative inflammatory bronchiolitis limited to the mid-bronchiole airway, which may be responsive to corticosteroid therapy and macrolide therapy, and fibrotic constrictive bronchiolitis, which is not responsive.

\section{Recent advances}

Several recent studies examining various aspects of constrictive bronchiolitis have been published in the last two years and are outlined here. In particular, intermittent reports of toxic fume constrictive bronchiolitis continue to be published; for example, as mentioned above, mustard gas exposure as a cause has recently been reported [2].

It has also been revealed that rheumatoid-arthritisrelated constrictive bronchiolitis is not as rare as previously thought, but continues to be poorly responsive to therapy and has a poor prognosis. In a 2009 report from France of 25 individuals with rheumatoid arthritis and obliterative bronchiolitis, most had severe airflow obstruction, often with an $\mathrm{FEV}_{1}$ of less than one liter, and did not improve when treated with corticosteroids [3]. The prognosis was poor: chronic respiratory failure occurred in $40 \%$ of patients and four died.

Immunological-disease-related constrictive bronchiolitis also continues to be reported. In a 2009 report from the 
Mayo Clinic, three additional patients with paraneoplastic autoimmune multi-organ syndrome developed constrictive bronchiolitis [4]. The three individuals had very severe airflow obstruction, with $\mathrm{FEV}_{1}$ values of much less than one liter, and the prognosis was poor. Two of them died and the third has stable chronic respiratory failure.

Swyer-James-MacLeod syndrome with unilateral constrictive bronchiolitis has always been considered rare, and now is no longer considered congenital in origin, but caused by a respiratory infection occurring during infancy and diagnosed during teenage years or later [5]. The respiratory infections during infancy include measles, whooping cough, tuberculosis, Mycoplasma pneumonia, influenza $\mathrm{A}$, and adenovirus types 3,7 , and $21[5]$.

HRCT scans continue to be important and valuable in establishing the diagnosis of constrictive bronchiolitis in all clinical settings. In a 2008 report from the Mayo Clinic describing 29 patients, mosaic perfusion and air trapping was seen in HRCT scans of all patients, whereas chest X-rays were normal in one-third and showed hyperinflation in just over one-half [6]. It is important to obtain both inspiratory and expiratory HRCT images in order to help visualize these characteristic abnormalities. In this group of 29 patients, shortness of breath occurred in all patients and cough in less than $20 \%$. The prognosis was poor: one patient improved, several stabilized, and four died.

Reports regarding constrictive bronchiolitis after hematopoietic stem cell transplantation continue to be published $[7,8]$. Chronic GVHD, which occurs in 30$70 \%$ of allogeneic transplant recipients, is the major risk factor. The prognosis is very poor after development of this fixed airway lesion, with a survival rate of $13 \%$ at five years [7]. The treatment of constrictive bronchiolitis is similar to that of chronic GVHD - high-dose corticosteroids and augmentation of immunosuppressive therapy, usually consisting of cyclosporine or tacrolimus. Azathioprine has also been used. The treatment is continued for 3-12 months, although 9 months is probably sufficient [8]. Anti-tumor necrosis factor agents have not yet been proven effective. Macrolides in the form of azithromycin (250 mg three times weekly) may be helpful for the bronchiole inflammatory component. High-dose inhaled corticosteroid therapy may be helpful; however, the agent is blocked by obliterated bronchioles and the high dose required may produce systemic adverse reactions. A randomized study has not yet been performed. Extracorporeal photopheresis therapy for treatment of GVHD and constrictive bronchiolitis may be helpful in these patients [8]. The prognosis continues to be poor as less than $20 \%$ of patients improve and $65 \%$ die within three years of the diagnosis of constrictive bronchiolitis regardless of therapies instituted, although lung transplantation can be considered in select patients [7].

Constrictive bronchiolitis in lung transplant recipients continues to plague surgeons and patients, and is the main obstacle to long-term survival. Reports are published on a monthly basis, most of which confirm previous findings and treatment results. Gastroesophageal reflux disease is a common finding among lung transplant recipients. Among 59 lung recipients, 53 (90\%) had reflux, of which $65 \%$ had abnormal acid reflux and $27 \%$ had non-acid reflux [9]. By using esophageal impedance, it was found that non-acid reflux may be a cause of constrictive bronchiolitis [9]. If nonacid reflux plays a role in lung transplant recipients, it may be that the reflux leads to an alloimmune response that causes the syndrome.

The six-minute walk test may be a useful tool for assessing prognosis in lung transplant recipients who have developed bronchiolitis obliterans syndrome. In a 2009 study, patients who walked farther than 330 meters had a median survival of 1178 days compared to 263 days for lung recipients who walked less [10].

Tacrolimus is an important immunosuppressant medication for lung transplant recipients. Compared to cyclosporine, patients treated with tacrolimus appear to have similar survival and fewer acute rejection episodes [11]. This study also revealed that tacrolimus-treated patients may have a lower risk of constrictive bronchiolitis, as this agent may decrease the occurrence of chronic rejection [11]. However, some data regarding tacrolimus and cyclosporine are conflicting, and there is no consensus as to which is better. Studies showing the inferiority of cyclosporine did not use the preferred method of monitoring trough levels. In addition, some reports have suggested that compliance with tacrolimus appeared to be better than with cyclosporine. Both tacrolimus and cyclosporine have significant toxicity and are associated with increased risk of opportunistic infections, hypertension, and renal insufficiency. Newonset diabetes after transplantation appears to be more common in lung transplant recipients treated with tacrolimus.

In an immune suppression study of 120 consecutive lung transplant recipients, the combination of tacrolimus and azathioprine was similar to cyclosporine and mycophenolate mofetil with regard to efficacy and frequency of adverse reactions. [12]. The survival and rate of 
bronchiolitis obliterans did not differ between the two regimens when combined with interleukin-2 induction therapy. Overall, studies in lung recipients have not consistently identified one immunosupression regimen to be superior to another and it is not clear that any have a significant impact on the long-term development of constrictive bronchiolitis.

Extracorporeal photopheresis is the removal of a patient's blood and its separation into leukocyteenriched and leukocyte-depleted blood components. The buffy coat of leukocytes is exposed to ultraviolet light in the presence of 8-methoxypsoralen in a photoactivation chamber. Several events take place in the chamber, including leukocyte apoptosis, changes in dendritic cell populations, production of cytokines, and induction of regulatory T cells [13]. Extracorporeal photopheresis is an accepted therapy for prophylaxis and treatment of acute cellular rejection in heart transplantation. Among 60 lung transplant recipients, a 2009 study showed that extracorporeal photopheresis treatment decreased the rate of $\mathrm{FEV}_{1}$ decline and the $\mathrm{FEV}_{1}$ improved in $25 \%$ of patients [13]. Extracorporeal photopheresis was associated with a significant reduction in the rate of decline in lung function, which was sustained over 6 and 12-month periods [13]. Large, randomized clinical studies are needed to confirm these results.

Macrolide antibiotics, such as azithromycin $(250 \mathrm{mg}$, three times weekly), can be effective treatment for chronic inflammatory lung diseases such as bronchiectasis, cystic fibrosis, and BOOP. A study of lungtransplant-related BOS indicated that patients may be classed as one of two phenotypes, one that shows early lavage neutrophils and may be responsive to azithromycin, and another without neutrophil inflammation that is not responsive [14]. This is consistent with the knowledge that constrictive bronchiolitis is a fibrotic process that is not responsive to macrolides, whereas inflammatory bronchiolar lesions are responsive to macrolides. As this was a study of BOS and not a histological diagnosis, the macrolide-responsive patients may have had inflammatory bronchiolitis while the nonresponsive patients may have had fibrotic constrictive bronchiolitis. An additional important finding about azithromycin is that it may reduce gastroesophageal reflux in lung transplant recipients, further decreasing the risk of constrictive bronchiolitis [14].

\section{Implications for clinical practice}

These recent publications on constrictive bronchiolitis have several implications. First, HRCT scans continue to be very helpful for the diagnosis of constrictive bronchiolitis in an individual with new onset airflow obstruction, early inspiratory crackles, and a normal chest X-ray. Second, constrictive bronchiolitis should be considered in a patient with rheumatoid arthritis who develops shortness of breath. The diagnosis can be established by obtaining an expiratory HRCT scan. Third, the Swyer-James-MacLeod syndrome is not a congenital disorder but constrictive bronchiolitis that is detected in young adults caused by a viral or bacterial infection during infancy. Fourth, the six-minute walk test may be a useful test for monitoring lung transplant recipients with BOS. Fifth, although used since the 1990s, tacrolimus appears to continue to be an important organ-transplant immune suppression medication. Sixth, extracorporeal photopheresis may be effective management for constrictive bronchiolitis in some patients. Finally, azithromycin may be effective in a select group of lung transplant recipients for prevention of BOS.

\section{Abbreviations}

BOOP, bronchiolitis obliterans organizing pneumonia; BOS, bronchiolitis obliterans syndrome; CT, computed tomography; $\mathrm{FEV}_{1}$, forced expired volume in 1 second; GVHD, graft-versus-host disease; HRCT, high-resolution chest CT.

\section{Competing interests}

The author declares that he has no competing interests.

\section{References}

I. Epler GR: Constrictive bronchiolitis obliterans: the fibrotic airway disorder. Expert Rev Resp Med 2007, I:I39-47.

2. Rowell M, Kehe K, Balszuweit F, Thiermann H: The chronic effects of sulfur mustard exposure. Toxicology 2009, 263:9-II.

3. Devouassoux G, Cottin V, Lioté H, Marchand E, Frachon I, Schuller A, Béjui-Thivolet F, Cordier JF: Characterisation of severe obliterative bronchiolitis in rheumatoid arthritis. Eur Respir J 2009, 33:|053-6|.

FI000 Factor 3.0 Recommended Evaluated by Gary Epler 26 Mar 2010

4. Maldonado F, Pittelkow MR, Ryu JH: Constrictive bronchiolitis associated with paraneoplastic autoimmune multi-organ syndrome. Respirology 2009, | 4:129-33.

5. Sulaiman A, Cavaille A, Vaunois B, Tiffet O: Swyer-James-MacLeod syndrome; repeated chest drainages in a patient misdiagnosed with pneumothorax. Interact Cardiovasc Thorac Surg 2009, 8:482-4.

6. Parambil JG, Yi ES, Ryu JH: Obstructive bronchiolar disease identified by CT in the non-transplant population: analysis of 29 consecutive cases. Respirology 2009, 14:443-8.

FI000 Factor 3.0 Recommended Evaluated by Gary Epler 26 Mar 2010

7. Williams KM, Chien JW, Gladwin MT, Pavletic SZ: Bronchiolitis obliterans after allogeneic hematopoietic stem cell transplantation. JAMA 2009, 302:306-I4.

8. Pandya CM, Soubani AO: Bronchiolitis obliterans following hematopoietic stem cell transplantation: a clinical update. Clin Transplant 2009, [Epub ahead of print]. 
9. King BJ, lyer H, Leidi AA, Carby MR: Gastroesophageal reflux in bronchiolitis obliterans syndrome: a new perspective. J Heart Lung Transplant 2009, 28:870-5.

10. Nathan SD, Shlobin OA, Reese E, Ahmad S, Fregoso M, Athale C, Barnett SD: Prognostic value of the $6 \mathrm{~min}$ walk test in bronchiolitis obliterans syndrome. Respir Med 2009, 103:|816-21.

II. Fan Y, Xiao YB, Weng YG: Tacrolimus versus cyclosporine for adult lung transplant recipients: a meta-analysis. Transplant Proc 2009, 4I:|82|-4.

12. Celik MR, Lederer DJ, Wilt J, Eser D, Bacchetta M, D'Ovidio F, Sonett JR, Arcasoy SM: Tacrolimus and azathioprine versus cyclosporine and mycophenolate mofetil after lung transplantation: a retrospective cohort study. J Heart Lung Transplant 2009, 28:697-703.
13. Morrell MR, Despotis G], Lublin DM, Patterson GA, Trulock EP, Hachem RR: The Efficacy of photopheresis for bronchiolitis obliterans syndrome after lung transplantation. J Heart Lung Transplant 2009, 29:424-31.

FI000 Factor 3.0 Recommended

Evaluated by Gary Epler 26 Mar 2010

14. Verleden GM, Vos R, De Vleeschauwer SI, Willems-Widyastuti A, Verleden SE, Dupont LJ, Van Raemdonck DE, Vanaudenaerde BM: Obliterative bronchiolitis following lung transplantation: from old to new concepts? Transpl Int 2009, 22:77I-9.

FI000 Factor 3.0 Recommended

Evaluated by Gary Epler 26 Mar 2010 